\title{
Meckel syndrome: what are the minimum diagnostic criteria?
}

\author{
C Wright, R Healicon, C English, J Burn
}

\begin{abstract}
Two sibs are described, the first of whom presented the classic Meckel syndrome triad of encephalocele, postaxial polydactyly, and characteristic renal cystic changes. The second sib had none of these abnormalities, but did show urethral atresia and preaxial polydactyly, two features previously described in some patients with Meckel syndrome. The two cases illustrate both the wide phenotypic spectrum of Meckel syndrome and the difficulty of attempting to define minimum diagnostic criteria for the disorder. The clinical implications arising from this problem are discussed.
\end{abstract}

( $($ Med Genet 1994;31:482-485)

The recessively inherited disorder known as Meckel syndrome is characterised typically by polydactyly, cystic kidneys, and a central nervous system malformation (usually occipital encephalocele) ${ }^{1-3}$ However, studies of families in which the proband had at least two of these three features showed that there is wide phenotypic variation within the disorder. ${ }^{4-6}$ Other associated abnormalities included cleft lip/palate, anophthalmia/microphthalmia, ductal plate malformation of the liver, and cardiac and genital anomalies, but some sibs only possessed one of the three classical abnormalities. This has prompted a number of authors to address the problem of what should be considered as minimum diagnostic criteria for Meckel syndrome.

The pattern of renal cystic change typically encountered is characteristic of the disorder ${ }^{7}$ and it has been proposed that a diagnosis of Meckel syndrome cannot be made in the absence of these changes. ${ }^{56}$ This paper describes two sibs, one with features of classical Meckel syndrome, the other with preaxial polydactyly and obstructive uropathy (secondary to urethral atresia) but without typical Meckel-type renal cystic change or other typical features of the disorder. The possible significance of these findings with respect to establishing diagnostic criteria for this syndrome is considered.

\section{Case reports}

The parents are white and unrelated and their first born was a female showing no congenital malformations. An ultrasound scan in the second pregnancy showed that the fetus (the proband) had multiple abnormalities, and the pregnancy was terminated at 19 weeks. The mother was aged 30 years at this time and the father 29 years. This fetus (fig 1) had microcephaly with a large occipital encephalocele, postaxial polydactyly of both hands, and gross distension of the abdomen owing to massive bilateral renal enlargement. Histologically the kidneys showed typical Meckel-type cystic changes, and there was a ductal plate malformation of the liver (fig 2). Chromosome analysis was normal.

Ultrasound scans during the third pregnancy showed oligohydramnios and a cystic mass within the abdomen of the fetus accompanied by bilateral hydronephrosis. The fetus died in utero and labour was induced. On external examination there was early maceration, and body measurements were those of 22 to 23 weeks' gestation (in keeping with the date of the LMP). The mouth was wide with full lips, the nose was rather flat, and there was micrognathia (fig 3). The left ear was posteriorly rotated and low set. The cranium and head circumference were normal; in particular there was no evidence of encephalocele. The left foot showed preaxial polydactyly, with three extra digits arising from the medial aspect of the foot (fig 4); on $x$ ray all had three phalanges but only the lateral two had metatarsal bones. The other three limbs were normal and there was no bowing of the long bones.

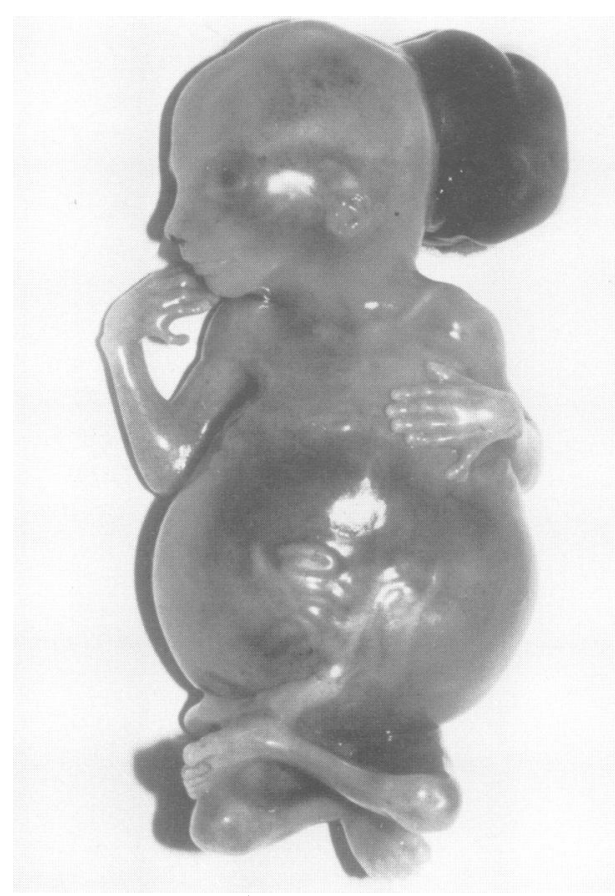

Figure 1 First sib, showing occipital encephalocele, postaxial polydactyly of upper limbs, and abdominal distension owing to massive renal enlargement. 

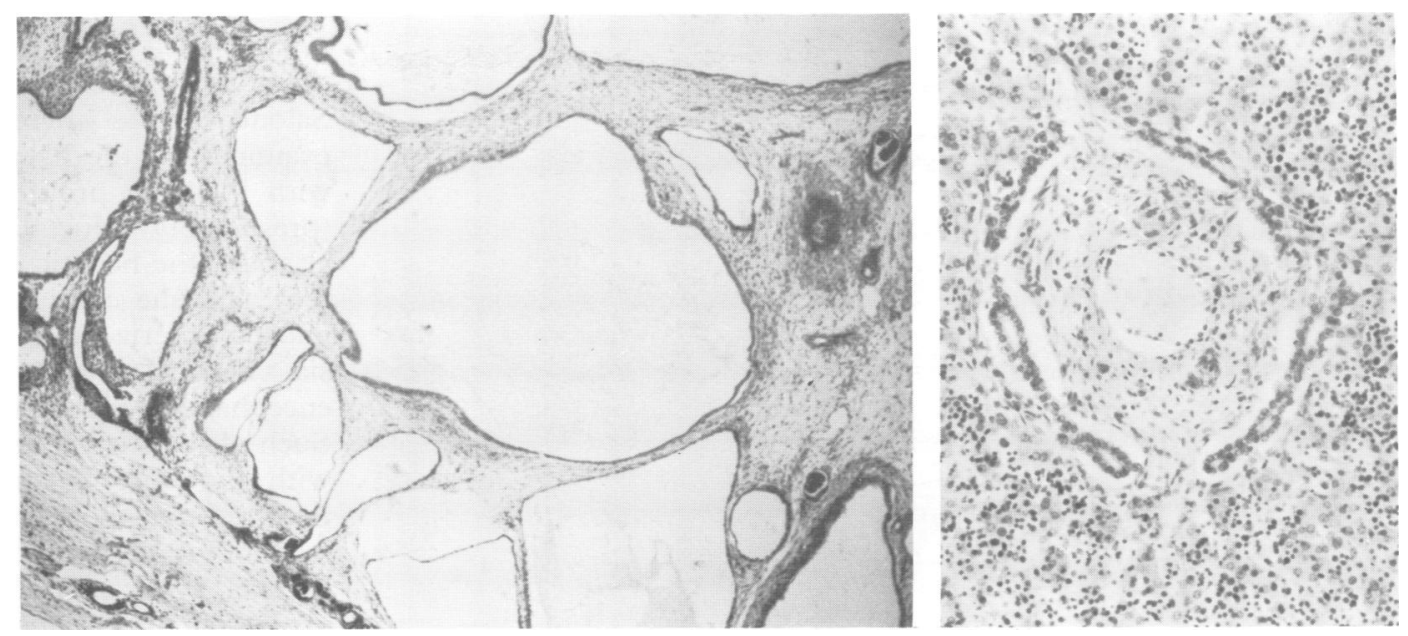

Figure 2 First sib. (Left) Histology of kidney in subcapsular area showing numerous cortical cysts. (Right) Histology of liver showing persistence of periportal bilaminar biliary cylinders (ductal plate malformation).

The urethra was atretic just distal to its origin from the bladder, which was massively dilated; there was associated bilateral hydroureter and hydronephrosis (fig 5). The renal parenchyma of both kidneys showed diffuse thinning and multiple subcapsular cysts up to $0.5 \mathrm{~mm}$ in diameter. Histologically there was general preservation of the renal architecture (fig 6). The subcapsular cysts were predominantly at the level of Bowman's capsule (glomerular cysts) and there was marked dilatation of the collecting ducts within both the medulla and cortex associated with focal increase in medullary connective tissue. However, cartilage, so called primitive ducts, and other features of renal dysplasia ${ }^{7}$ were not observed.

The brain was of normal weight $(75 \mathrm{~g})$ and on macroscopic and histological examination showed no evidence of congenital anomaly.

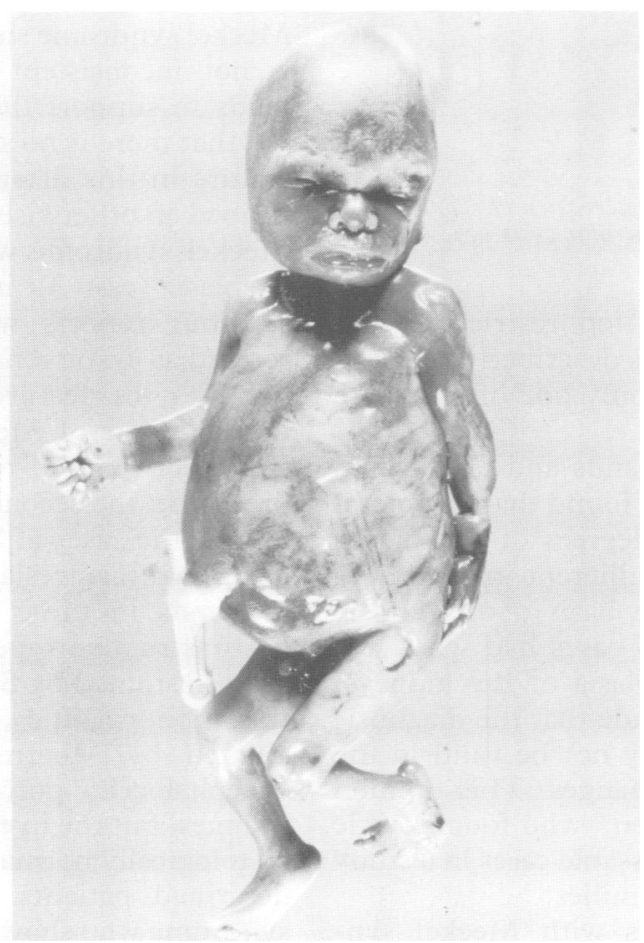

Figure 3 Second sib, showing rather full lips, flattened nose, distended abdomen, and preaxial polydactyly of the left foot.
Specifically there was no neural tube defect or ventriculomegaly. Except for pulmonary hypoplasia (lung:body weight ratio $1 \cdot 25 \%$ ) there were no other observable abnormalities at necropsy. The liver was histologically normal; there was no ductal plate malformation. Chromosome analysis of cardiac blood showed an apparently normal 46,XY karyotype.

\section{Discussion}

Faced with the phenotypic diversity presented by patients with Meckel syndrome, previous authors have attempted to set down minimum criteria for the diagnosis of the disorder. Hsia et $a l^{2}$ and Mecke and Passarge ${ }^{3}$ suggested that at least two of the classic triad of occipital encephalocele, polydactyly, and cystic kidneys should be present. However, Fitch and Pinsky ${ }^{8}$



Figure 4 Second sib: preaxial polydactyly of left foot. 


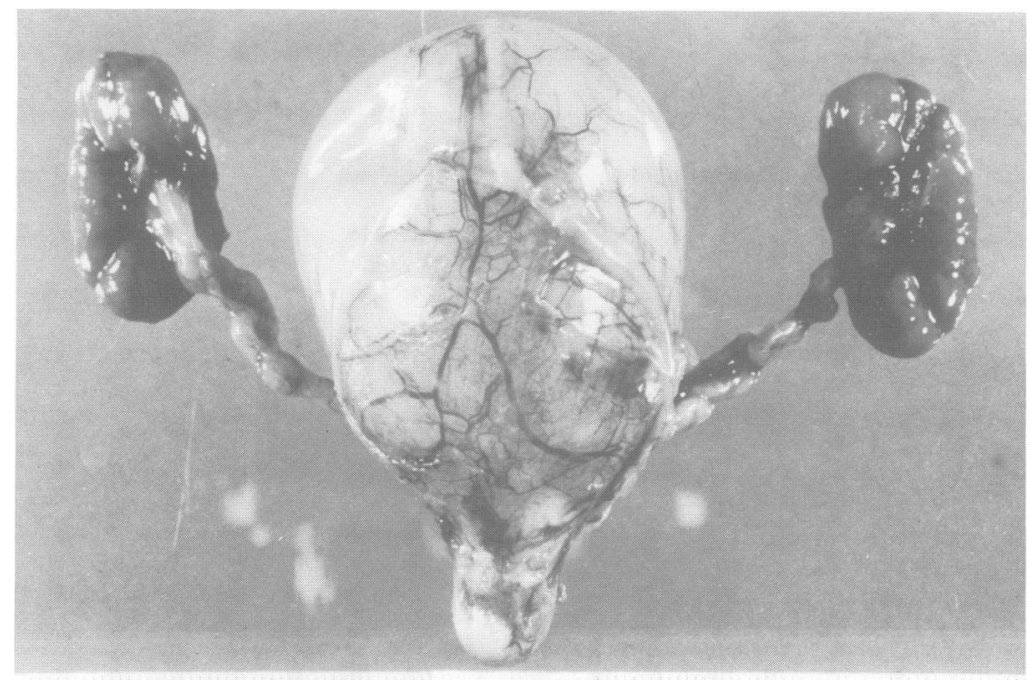

A $156 / 92$

Figure 5 Second sib: kidneys and urinary tract showing massively dilated bladder and tortuous, distended ureters. Note blind ending proximal urethra and normal sized kidneys.

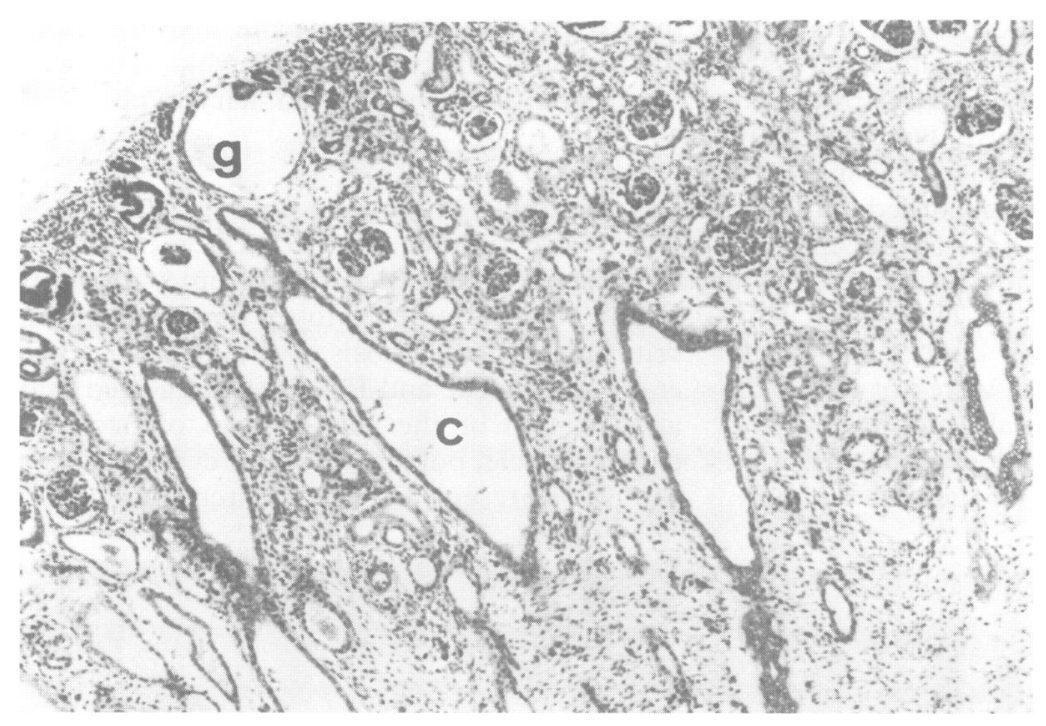

Figure 6 Second sib: histology of kidney showing general preservation of renal architecture, dilated collecting ducts $(\mathrm{c})$, and occasional glomerular cysts $(\mathrm{g})$.

felt such criteria to be too restrictive, and noted that one of the cases described by Hsia et $a l^{2}$ appeared to possess only one of the three features (cystic kidneys).

In a study of 27 probands and 38 affected sibs, Fraser and Lytwyn ${ }^{5}$ found that in general the sibs showed lesser degrees of malformation, reflecting the bias inherent in the selection of probands on the basis of the classic criteria. However, all 65 cases had what was described as cystic dysplasia of the kidneys, which led them to conclude that the diagnosis of Meckel syndrome may not be valid in the absence of such renal changes. This opinion was supported by Salonen, ${ }^{6}$ who found cystic renal dysplasia in all assessable cases in a study of 67 patients from 48 families.

The livers of patients with Meckel syndrome typically show retarded development of the intrahepatic biliary tree with inappropriate retention of the primitive ductal plate, often accompanied by excess portal tract connective tissue (ductal plate malformation). ${ }^{9}$ In all 41 of Salonen's cases where tissue was available for examination, the liver showed portal fibrosis with "ductal proliferation and dilatation" (presumably ductal plate malformation, althouth the hepatic changes are not illustrated), and she considered that minimum diagnostic criteria should be cystic renal dysplasia, hepatic portal fibrosis, and occipital encephalocele or other CNS malformation. ${ }^{6}$ Such strict criteria are, however, at variance with the observation made by Fraser and Lytwyn $^{5}$ that there was no brain abnormality in $18 \%$ of the sibs they considered to have Meckel syndrome, and some authors have expressed disagreement with the idea of an obligatory abnormality. ${ }^{10}$

The first of the sibs described here shows the classic triad of anomalies, and also fulfils Salonen's criteria. However, it is likely that without knowledge of this sib, the possibility of Meckel syndrome would not have been entertained in the second case. There were certainly renal cystic changes, but these were confined to the subcapsular parts of the kidneys which were otherwise architecturally normal, and were consistent with the effects of urinary obstruction owing to the urethral atresia (Potter type IV cysts). ${ }^{11}$ The kidneys did not show the features characteristically associated with Meckel syndrome, that is, numerous, rather rounded cysts of varying size (some of the level of glomeruli), with no discernible corticomedullary junction. ${ }^{7}$ The second sib did not show any CNS or hepatic abnormalities, and although there was polydactyly, this was preaxial and confined to one lower limb, while polydactyly in Meckel syndrome is typically postaxial and bilateral. ${ }^{56}$

However, closer study of reported cases of Meckel syndrome suggests that this phenotype is not inconsistent with the diagnosis, and tends to support the contention of Lowry et $a l^{10}$ that there is no such thing as an obligatory feature in this disorder: Moerman et al ${ }^{12} \mathrm{de}-$ scribed an otherwise apparently typical case of Meckel syndrome which did not show cystic dysplasia (case 3); it is not in fact clear from previous reports whether the changes described as cystic renal dysplasia always correspond to the features now recognised to be characteristic of Meckel syndrome, or are in fact other types of renal cystic change such as present in the second sib above. For example, four of the cases of Salonen ${ }^{6}$ were reported to have urethral atresia, and it is conceivable that the cysts in these cases may have been of postobstructive type. Although all assessable cases examined by Salonen ${ }^{6}$ had hepatic portal tract abnormalities, one case (case 5) documented by Moerman et $a l^{12}$ had occipital encephalocele, polydactyly, and renal cystic changes, but the liver was macroscopically and histologically normal. Finally, Majewski et al ${ }^{13}$ described patients with features of Meckel syndrome who showed pre- (rather than post-) axial polydactyly.

The second of the sibs reported here shows features which overlap with the entity reported 
as distal obstructive uropathy with polydactyly: Halal ${ }^{14}$ described two unrelated stillborn infants with hydronephrosis, hydroureter, and bladder dilatation secondary to urethral obstruction (posterior urethral valves in one case, uncertain urethral pathology in the second), together with postaxial polydactyly. It is possible that the pattern of malformations shown by the second sib of the family reported here represents a disorder other than Meckel syndrome, either as a simple coincidence or, just possibly, as the differing unbalanced phenotypes of an unrecognised translocation. It seems on balance more likely, however, that they represent extremes of the spectrum of abnormalities occurring in Meckel syndrome. The breadth of the phenotypic spectrum creates problems in genetic counselling and prenatal diagnosis. The possibility of this recessive disorder should be borne in mind when considering a proband with any of the key features, even in apparent isolation. The efficacy of second trimester ultrasonography in subsequent pregnancies is reduced since the absence of an encephalocele, postaxial polydactyly, and renal dysplasia is not completely reassuring. It is important that tissue for molecular genetic analysis is stored from all such families as a resource to allow the development of a more reliable diagnostic test.
1 Opitz JM, Howe JJ. The Meckel syndrome (dysencephalia splanchnocystica, the Gruber syndrome). Birth Defects 1969;5:167-79.

2 Hsia YE, Bratu M, Herbordt A. Genetics of the Meckel syndrome (dysencephalia splanchnocystica). Pediatrics 971;48:237-47.

3 Mecke S, Passarge E. Encephalocele, polycystic kidneys and polydactyly as an autosomal recessive trait simulating certain other disorders: the Meckel syndrome. Ann Genet (Paris) 1971;14:97-103.

4 Seller MJ. Phenotypic variation in Meckel syndrome. Clin Genet 1981;20:74-7.

5 Fraser FC, Lytwyn A. Spectrum of anomalies in the Meckel syndrome or: "Maybe there is a malformation syndrome with at least one constant anomaly". Am f Med Genet 1981;9:67-73

6 Salonen R. The Meckel syndrome: clinicopathological findings in 67 patients. Am $\mathcal{F}$ Med Genet 1984;18:671-89.

7 Rapola J. The kidneys and urinary tract. In: Wigglesworth JS, Singer DB, eds. Textbook of fetal and perinatal pathoJogy. Binger DB, eds. Textbook of fetal and permatal patho-
logy: Blackwell Scientific Publications, 1991:1109-43.

8 Fitch N, Pinsky L. The Meckel syndrome with limited expression in relatives. Clin Genet 1973;4:33-7.

9 Blankenberg TA, Ruebner BH, Ellis WG, Bernstein J, Dimmick JE. Pathology of renal hepatic anomalies in Meckel's syndrome. Am $\mathcal{F}$ Med Genet (Supp 1) 1987;3: 395-410.

10 Lowry RB, Hill RH, Tischler B. Survival and spectrum of anomalies in the Meckel syndrome. Am $7 \mathrm{Med}$ Genet 1983;14:417-21.

11 Zerres K, Volpel MC, Weiss H. Cystic kidneys. Genetics, pathologic anatomy, clinical picture and prenatal diagpathologic anatomy, clinical pict
nosis. Hum Genet 1984;68:104-35.

12 Moerman P, Verbeken E, Fryns JP, Goddeeris P, Lauweryns JM. The Meckel syndrome. Pathological and cytogenetic observations in eight cases. Hum Genet 1982;62:240-5

13 Majewski F, Stoss H, Goecke T, Kemperdick H. Are bowing of long tubular bones and preaxial polydactyly signs of the Meckel syndrome? Hum Genet 1983;65:12533.

14 Halal F. Distal obstructive uropathy with polydactyly: a new syndrome? Am 7 Med Genet 1986;24:753-57. 